\title{
Cheshire: A Design Framework for Alternate Reality Games
}

\author{
Alejandro I. Baltra* \\ Universidad Técnica Federico Santa María, Valparaíso, Chile \\ abaltra@inf.utfsm.cl
}

\begin{abstract}
Alternate Reality Games (ARG) have come very far in the last ten years. Great works by the likes of Steve Peters, Christy Dena and Jim Miller have pushed the genre forward, but it still lacks some of the tools created for other types of games. The design process for ARGs is, in the best case scenario, complicated. Given the enormous amount of media these kind of games use, things can escalate from complex to utterly impossible in the blink of an eye and sadly, there are currently no tools to help solve this problem or lower its impact in the finished game. This paper proposes Cheshire, a framework to enable categorization of games and alignment of these with defined sets of patterns previously detected in the design of the experience. This tool will help maintain the generated experience as loyal to the original concept as possible, and graphically represent which elements will support it in the best manner.
\end{abstract}

Keywords: Alternate Reality Games, ARG, Game Design, Digital Narrative.

\section{Introduction}

Alternate Reality Games have gained much notoriety in the latest years due to their impact as promotional tools for other types of media, but this doesn't mean this is the only way such genre can be enjoyed. Many efforts have been made towards pushing this particular kind of games forward, but the many layers of complexity that crafting this kind of experiences garners makes the task of designing such games a very real challenge.

In this paper we attempt to solve such issue by presenting Cheshire, a framework that allows the definition of core elements and design patterns a given ARG has to align with in order to deliver the kind of experience it's designed to provide.

\section{The ARG Design Problem}

ARG design is, at best, complicated. The process, even though it has many similarities with regular game design, tends to tilt towards the more difficult part of the spectrum due to the complexity of the media and delivery channels the designers

\footnotetext{
This work was partially supported by FONDEF through grant D08i1155 (project ContentCompass).
} 
have to keep in mind. Besides this obvious difficulty, there are no tools specifically created to support designers in this task. Currently, the tools used aren't anymore customized than Gantt charts and workflows.

The problems mentioned can be pointed as one of the main causes of the long development times ARGs can represent and, to a given extent, to several games disappearing before seeing the light of day (a situation know as meltdown [1]).

\section{The Design Framework}

\subsection{Core Elements}

First of all, a set of core interactions and elements has to be described. To construct a proper framework, we'll base our model on MDA [2], where the design process is modeled using three different stages. Even though this framework can't be used directly on ARGs (given MDA's focus on predefining dynamics), the idea of a layered information flow proves to be very useful.

It's important to note that ARGs are generally social experiences, but single player experiences aren't out of the question. Only taking into account the social aspect of it and relegating the flow and gaming sensation would transform it in nothing short of a social network or discussion group. The proper introduction of these gaming elements to the public allows for flow and momentum control, modeling the experience. The most basic of these elements is the narreme (a term coined by Lev Manovich [5]), which contains important information to advance in the game. This new information generates pieces of media that work as puzzles, obstacles and challenges for the players to overcome, adding a gameplay layer. Finally, these obstacles are overcome, generally in a social manner, completely out of the control of the designer in an emerging fashion; this means the designers can only present the problem, the way to solve it falls on the players' shoulders.

Once the players have passed the challenge presented by a particular piece of media, a new narreme is introduced, taking the role of gratification as well as motivation to keep on advancing the game.

Now that the basic elements and their relations have been described, we must mention the behaviors they elicit. These behaviors are:

- Sense of Movement: When players start the game or overcome a high impact obstacle, they get a narreme. This element holds two functions, first to gratify the player and second to kick-start the next story element, allowing for the development of characters, situations and events.

- Obstacles and Challenge: Media by itself has no meaning beyond presenting information; it's through the existence of challenge that players feel compelled to keep playing. Through media this obstacles are presented to the participants.

- Emerging Dynamics: Since ARGs are generally social, the obstacles and challenges must be designed with this in mind. For that reason, the way players solve a problem is out of the designers' control, they can only define a starting point (problem) and an ending (solution).

These elements and their relations can be seen on figure 1 . 


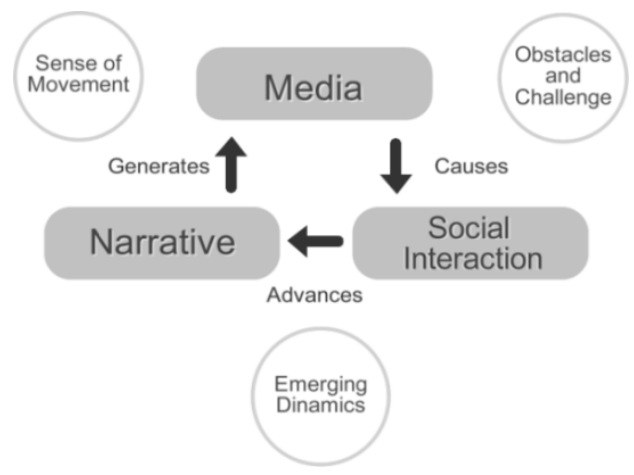

Fig. 1. Core elements and the behaviors they elicit

It's important to notice that the media can be presented in two mayor ways, it can be unidirectional, meaning it only goes from the designers to the players or vice versa without an automatic response; this type will be called passive. On the other hand, there's media that looks for direct responses to a given interaction by the players. This type will be called active.

Each of these two types of media generates a different impact on the general flow of the game. The active media causes a greater apparition of narrative elements, since puzzles in ARGs tend to require lengthy analysis, the fast nature of this media makes it better suited to deliver story elements that advance the plot and don't present big obstacles. On the other hand, passive media gives players enough time to think about the challenges presented and correctly solve the puzzles.

These relationships are displayed on figure 2 .

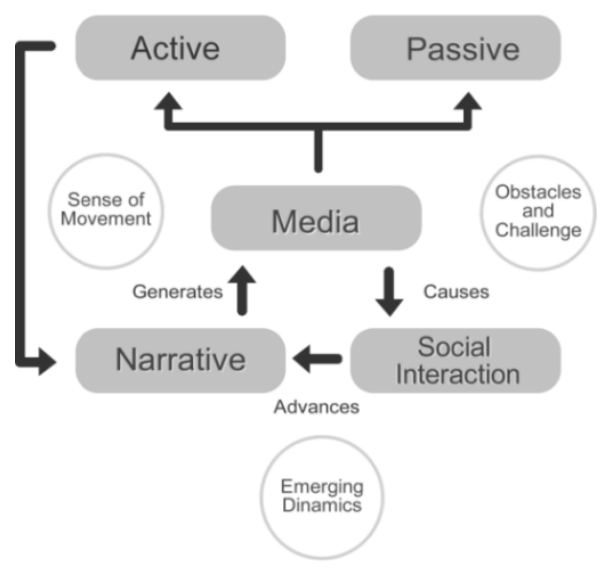

Fig. 2. Differentiation of types of media and their impact in the game flow 


\subsection{Patterns and Alignment}

With the core elements defined, it's possible to align them with a defined set of design patterns. This alignment helps give the designers a general guideline of what patterns to use in the design process to achieve the kind of game they set out to create.

The patterns used were the ones compiled by Christy Dena in [4], which are in turned based on the work displayed in [3]. Even though there are many more game design patterns out there, the work of compiling which ones are particularly useful for ARGs has been done previously and we'll use them as an already analyzed element in our framework.

These patterns are grouped in four categories:

- Collaboration: An event or situation inside the game requires interaction and cooperation between players with different skills or, in other case, in a common problem that requires a large number of people working in tandem.

- Group Activities: In some games, players do things in rather static groups.

- Stimulated Social Interaction: Games played face to face tend to have a high degree of social interaction, be it required by the game or not.

- Competition: Despite social interaction tend to be associated with players doing things together; the road to such interactions tends to be in most cases, paved with conflict and competition amongst them.

One important thing to note is that, given the similar characteristics that Collaboration and Group Activities share, we'll merge them, encompassing all patterns of both categories. Why is it useful to merge them? Having only three axes to use in the framework makes the creation of a graphical representation much easier, since we can resemble a Cartesian model with three planes ${ }^{1}$.

In this three-plane representation and with the previously described core elements centered on the junction of the three categories, it's possible to orderly list the patterns in each category around the core elements, properly giving a guideline as to which pattern must be more emphasized when creating the elements needed for the game.

Each of the patterns was placed based on their definition and their degree of alignment with each category (for example, Dynamic Alliances is centered in the Group Activities axis, while Conflict and Player Murder are much closer to Competition). In addition to the placement of the patterns, a color code was applied. Having properly aligning the colors with the axes (for a full color version, please refer to footnote 2), it's possible to classify a given game based on the patterns it uses and where in the color spectrum landed. How is this done? It's pretty simple, actually: the moment the design starts, the designers choose which category to focus on; once defined, we choose a specific color and, given the distance relation between the selected point in the spectrum and each given pattern, the emphasis each different pattern requires to achieve the a specific type of game is defined.

\footnotetext{
${ }^{1}$ For the sake of space, a full-color, high-definition graphical representation of the framework can be found in http://alumnos.inf.utfsm.cl/ abaltra/diag.png
} 


\section{Validation}

To illustrate the usefulness of Cheshire, two games were designed. The first one, built by a team of students from UTFSM ${ }^{2}$ and members of USMGames ${ }^{3}$ (Team A), was designed in the lapse of over four weeks and defined a game time of barely over a week. This team did not use Cheshire, only focused its' efforts using Gantt Charts and regular game design techniques.

On the other hand, a second game was designed, this time by a smaller team on a more constricted schedule (Team B). This time, the team used Cheshire to define which patterns to emphasize when crafting the experience and guide the design process. The design took little over two weeks and defined a game time of two weeks.

Both teams were formed by members of the same University with rather similar knowledge of game design and implementation; different topics were used for each game, but the basic literacy of each group on each given subject was roughly the same.

After both design processes ended, some differences are worth noting:

Table 1. Comparison of characteristics of both designed games

\begin{tabular}{|l|l|l|}
\cline { 2 - 3 } \multicolumn{1}{c|}{} & \multicolumn{1}{c|}{ Team A } & \multicolumn{1}{c|}{ Team B } \\
\hline Design Team & 2 people. & 1 person. \\
\hline Expected Players & Under 100. & Over 150. \\
\hline Planned game time & One week. & Two weeks. \\
\hline In-game routes & $\begin{array}{l}\text { One entry point with 5 } \\
\text { possible paths. All paths } \\
\text { must be traversed. }\end{array}$ & One lineal path. \\
\hline Actors & $\begin{array}{l}\text { None on-site, one } \\
\text { administrator sends data } \\
\text { to the payers. }\end{array}$ & $\begin{array}{l}\text { At least 4 on-site, one } \\
\text { administrator per site and } \\
\text { at least 4 users on each } \\
\text { web page. }\end{array}$ \\
\hline Digital Tools & $\begin{array}{l}\text { One website and one } \\
\text { mobile app. }\end{array}$ & $\begin{array}{l}\text { Two websites and at least } \\
\text { one mobile app. }\end{array}$ \\
\hline Design time & One week. & Two weeks. \\
\hline
\end{tabular}

Table 1 shows that the human effort invested by Team B was a lot less than that of Team A, even though the second game was of at least the same (if not higher) complexity.

A point to keep in mind, and that perhaps shows more clearly the contribution of Cheshire is that both games went in completely different directions by the end of their development. Team As' game drifted towards a treasure hunt, even though it was initially going to be a story-driven game while Team Bs‘ experience stayed close to its' core concept, a character driven adventure with light usage of geolocation.

\footnotetext{
${ }^{2}$ Universidad Técnica Federico Santa María.

${ }^{3}$ http: / / www . usmgames.cl
} 


\section{Conclusions and Future Work}

For anyone who's ever designed an ARG, it's clear that it's impossible to create an absolutely generic tool that carries the whole design process. The breadth of such process tends to be of such magnitude that a tool like that would only be useful for a particular game and not for many different experiences. However, the beginning of the experience can be aided by the use of Cheshire, as the proper selection of patterns gives the designers a very useful set of guidelines to which adhere their works to.

\section{References}

[1] IGDA Alternate Reality Games SIG, et al.: 2006 Alternate Reality Games. White Paper (2006), http://archives.igda.org/arg/whitepaper.html (last access: April 6, 2011)

[2] Hunicke, R., Leblanc, M., Zubek, R.: MDA: Formal Approach to Game Design and Game Research (2004), http: / / cs . northwestern. edu/ hunicke/pubs / MDA.pdf

[3] Bjork, S., Holopainen J.: Patterns in Game Design, 1st edn. Charles Rivers Media (December 2004)

[4] Dena, C.: Social Interaction Patterns in Alternate Reality Games v0.1 (June 2008), http: / /www. christydena.com/Primer/ARGDashboard_Interaction.h tml (last access: April 6, 2011)

[5] Manovich, L.: El Lenguaje de los Nuevos Medios de Comunicación/The Language of New Media, Paidos Iberica (2005) 